\title{
GNSS Reliability Testing in Signal-Degraded Scenario
}

\author{
A. Angrisano, C. Gioia, S. Gaglione, and G. del Core \\ "Parthenope" University of Naples, Centro Direzionale di Napoli Isola C4, 80143 Naples, Italy \\ Correspondence should be addressed to C. Gioia; ciro.gioia@uniparthenope.it \\ and S. Gaglione; salvatore.gaglione@uniparthenope.it
}

Received 25 October 2012; Revised 11 February 2013; Accepted 21 February 2013

Academic Editor: Sandro Radicella

Copyright (C) 2013 A. Angrisano et al. This is an open access article distributed under the Creative Commons Attribution License, which permits unrestricted use, distribution, and reproduction in any medium, provided the original work is properly cited.

Multiconstellation satellite navigation is critical in signal-degraded environments where signals are strongly corrupted. In this case, the use of a single GNSS system does not guarantee an accurate and continuous positioning. A possible approach to solve this problem is the use of multiconstellation receivers that provide additional measurements and allows robust reliability testing; in this work, a GPS/GLONASS combination is considered. In urban scenario, a modification of the classical RAIM technique is necessary taking into account frequent multiple blunders. The FDE schemes analysed are the "Observation Subset Testing," "Forward-Backward Method," and "Danish Method"; they are obtained by combining different basic statistical tests. The considered FDE methods are modified to optimize their behaviour in urban scenario. Specifically a preliminary check is implemented to screen out bad geometries. Moreover, a large blunder could cause multiple test failures; hence, a separability index is implemented to avoid the incorrect exclusion of blunder-free measurements. Testing the RAIM algorithms of GPS/GLONASS combination to verify the benefits relative to GPS only case is a main target of this work too. The performance of these methods is compared in terms of RMS and maximum error for the horizontal and vertical components of position and velocity.

\section{Introduction}

GNSS (Global Navigation Satellite Systems) are worldwide, all-weather navigation systems able to provide threedimensional position, velocity, and time synchronization to UTC (Coordinated Universal Time) scale $[1,2]$. The GPS system is the main GNSS and it is fully operational since almost two decades; in good visibility conditions ("open sky" scenario), GPS can provide a position accuracy of few meters for absolute positioning up to millimetre order for postprocessed relative positioning [2].

Satellite navigation in difficult scenarios (e.g., urban canyons, and mountainous areas) is more critical, because many GNSS signals are blocked or strongly degraded by natural and artificial obstacles; in these scenarios GPS only cannot guarantee an accurate and continuous positioning due to the lack of measurements and/or the presence of erroneous measurements. A possible way to fill this gap is the use of a GNSS multiconstellation receiver, considering the combined use of GPS with other GNSS such as Galileo, Beidou, and GLONASS.

The performance of the integrated system is increased in terms of

(i) continuity, directly related to satellite availability,

(ii) accuracy, enhanced by observation geometry improvement, and

(iii) integrity, as the increased availability improves the detection process of gross errors $[3,4]$.

Galileo currently has only four satellites in orbit, in the IOV (In-Orbit Validation) phase, while Beidou is currently in the development phase. The enhancement of the Russian space program has made GLONASS an ideal candidate to form a multiconstellation with GPS. The combined use of these two systems implies the estimation of a further unknown (in addition to navigation parameters), representing the 
TABLE 1: GPS/GLONASS differences.

\begin{tabular}{|c|c|c|c|}
\hline & Parameter & GPS & GLONASS \\
\hline \multirow{6}{*}{ Constellation } & Number of SV & 24 (expandable) & 24 \\
\hline & Orbital planes & 6 & 3 \\
\hline & Orbital altitude & $20200 \mathrm{~km}$ & $19100 \mathrm{~km}$ \\
\hline & Orbit inclination & $55^{\circ}$ & $64.8^{\circ}$ \\
\hline & Ground track period & 1 sidereal day & 8 sidereal days \\
\hline & Layout & Asymmetric & Symmetric \\
\hline \multirow{4}{*}{ Signal } & Carrier frequencies $(\mathrm{MHz})$ & $\begin{array}{l}1575.42 \\
1227.60\end{array}$ & $\begin{array}{c}1602+K * 0.5625 \\
1246+K * 0.4375\end{array}$ \\
\hline & Ranging code frequencies (MHz) & $\begin{array}{c}\text { C/A: } 1.023 \\
\text { L2C: } 1.023 \\
\text { P: } 10.23 \\
\text { M: } 10.23\end{array}$ & $\begin{array}{l}\text { C/A: } 0.511 \\
\text { P: } 5.11\end{array}$ \\
\hline & Multiple access schemes & CDMA & FDMA \\
\hline & Broadcast ephemerides & Keplerian & ECEF \\
\hline \multirow{2}{*}{ Reference } & Datum & WGS84 & PZ90.02 \\
\hline & Time scale & GPS time & GLONASS time \\
\hline
\end{tabular}

timescale offset between the considered systems, with the "sacrifice" of one measurement.

Integrity monitoring has a great importance in safetycritical operation, like air navigation, or in signal-degraded scenario, where solution could be unacceptably inaccurate.

Measurements in urban scenario are strongly affected by gross errors, degrading navigation solution; hence a quality check on the measurements, defined as RAIM, is important. The integrity of a navigation system is defined as the ability to provide timely warnings to users when the system should not be used [2, 5]. GPS and GLONASS provide integrity information to users into the navigation message, but this may be not timely enough for real-time application [5]; hence, additional tools have to be used. Two different approaches are possible to provide integrity. The first employs a network of ground stations to monitor GNSS signals, and the integrity information is transmitted to the users by a data link; typical examples of this mode are GBAS (Ground Based Augmentation System) and SBAS (Satellite Based Augmentation System). The second approach is the RAIM technique, which is based on self-consistency check on redundant measurements and so it is able to detect user-level errors as multipath or local interference, strongly degrading the navigation solution. The classical RAIM algorithms were developed for aviation; hence, they need to be redesigned for urban applications. In this context three well-known schemes, the "Observation Subset Testing," the "ForwardBackward Method," and the "Danish Method," are modified by implementing additional modules to screen out bad geometries and to identify the influence of a large blunder on error-free measurements.

\section{GNSS}

In this work GPS and GLONASS are considered because they are the only systems declared fully operational; they are similar for many aspects, such as the operational principle detailed in Section 2.1; however, they present some significant differences detailed in Section 2.2.

2.1. GPS GLONASS Common Aspects. GPS and GLONASS positioning is based on the one-way ranging technique: the time of travel of a signal, transmitted by a satellite, is measured and scaled by the speed of light to obtain the satellite-user distance, called pseudorange (PR), whose equation is $[1,5]$

$$
\rho=d+c \delta t_{u}+\varepsilon_{\rho}
$$

where $\rho$ is the PR measurement, $d$ is the geometric receiversatellite distance, $c \delta t_{u}$ is the receiver clock offset scaled by the speed of light $c$, and $\varepsilon_{\rho}$ contains the residual errors after atmospheric and satellite-related corrections.

Trilateration uses PR measurements to compute the navigation unknowns that are the tridimensional receiver coordinates and the receiver clock offset relative to the system time scale.

GNSS receivers are also able to obtain Doppler measurements, defined as the time derivative of observable phase $[1,2]$ and related to the relative motion between the receiver and satellites. Doppler observable is directly converted in a pseudorange rate whose equation is [2]

$$
\dot{\rho}=\dot{d}+c \dot{\delta} t_{u}+\varepsilon_{\dot{\rho}}
$$

where $\dot{\rho}$ is the PR rate measurement, $\dot{d}$ is the time derivative of the geometric distance receiver-satellite, $c \dot{\delta} t_{u}$ is the receiver clock drift (scaled by speed of light $c$ ), and $\varepsilon_{\dot{\rho}}$ is the residual errors after satellite-based corrections.

2.2. GPS GLONASS Differences. GPS and GLONASS are based on the same operating principle but they have several differences, which can be classified in terms of constellation, signal, and reference. The differences between the two systems are summarized in Table 1 and detailed in $[6,7]$. 
A comprehensive description of the aforesaid differences among GPS and GLONASS is provided in Angrisano 2010 [7].

For our scope the main difference is related to the different time scale adopted by the systems. GPS time is connected with UTC(USNO), the UTC maintained by US Naval Observatory; UTC scale is occasionally adjusted to one second to keep the scale close to the mean solar time. GPS time scale differs from UTC(USNO) of an integer number of seconds (called leap seconds, currently $16 \mathrm{~s}$ ); a further difference between GPS and UTC(USNO) time scale (typically less than $100 \mathrm{~ns}$ ), due to the different master clocks used, is broadcast to the users within the GPS navigation message. GLONASS time scale is connected to UTC(RU), the UTC maintained by Russia; it is corrected by leap seconds, according to the UTC adjustments, so the difference between these time scales is less than 1 millisecond and is broadcast in the GLONASS navigation message.

GPS and GLONASS time scales are connected by the following relation $[8,9]$ :

$$
t_{\mathrm{GPS}}=t_{\mathrm{GLO}}+\tau_{r}+\tau_{u}+\tau_{g} \text {, }
$$

where $\tau_{r}=t_{\mathrm{UTC}(\mathrm{RU})}-t_{\mathrm{GLO}}$ is broadcast within the GLONASS navigation message, $\tau_{u}=t_{\mathrm{UTC}(\mathrm{USNO})}-t_{\mathrm{UTC}(\mathrm{RU})}$ must be estimated, and $\tau_{g}=t_{\mathrm{GPS}}-t_{\mathrm{UTC} \text { (USNO) }}$ is broadcast in the GPS navigation message.

To perform the transformation (3), $\tau_{u}$ should be known, but this information is not provided in real time. An estimation of $\tau_{u}$ is broadcast as nonimmediate parameter in the GLONASS almanac [10], but does not take into account the intersystem hardware delay bias which is dependent on specific receiver [8]. Therefore, when GPS and GLONASS measurements are used together, $\tau_{u}$ is included in the estimation process as unknown.

2.3. Estimation of Navigation Parameters. In this work the adopted estimation technique is the weighted least squares (LS), which uses only a measurement model made up by simultaneous PR and PR rate observables.

Regarding PR, a set of equations like (1), after linearization around a nominal state, becomes

$$
\underline{\Delta \rho}=H_{\rho} * \underline{\Delta x}+\underline{\varepsilon} \rho,
$$

where $\Delta \rho$ is the difference between actual and predicted measurements, $H_{\rho}$ is the design matrix, $\underline{\varepsilon}_{\rho}$ is the residual error vector, and $\underline{\Delta x}$ is the state vector containing receiver coordinates and clock offset errors:

$$
\underline{\Delta x}=\left[\begin{array}{ll}
\underline{\Delta P}^{T} & \Delta\left(c \delta t_{u}^{\mathrm{GPS}}\right)
\end{array}\right]^{T} .
$$

If GPS and GLONASS measurements are used together, an additional unknown must be estimated, as explained in Section 2.2, and the state vector becomes

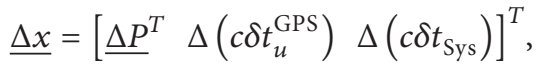

where $\Delta\left(c \delta t_{\text {Sys }}\right)$ is the offset between GPS and GLONASS time scales.
The measurement model of $\mathrm{PR}$ rate observable is

$$
\underline{\Delta \dot{\rho}}=H_{\dot{\rho}} * \underline{v}+\underline{\varepsilon} \dot{\rho}
$$

where $\Delta \dot{\rho}$ is the vector containing PR rate measurements, corrected for satellite motion, $H_{\dot{\rho}}$ is the design matrix, $\underline{\varepsilon}_{\dot{\rho}}$ is the residual error vector, and $\underline{v}$ is the velocity state vector:

$$
\underline{v}=\left[\begin{array}{ll}
\underline{V}^{T} & \dot{c} t_{u}^{\mathrm{GPS}}
\end{array}\right]^{T} .
$$

In this case the equations are linear for the unknowns, and the GPS/GLONASS combined use does not imply an additional unknown, because the drift between the system time scales is negligible.

For the PR measurement, the follow accuracy, dependent on satellite elevation, is assumed:

$$
\sigma_{\mathrm{PR}}^{2}=\sigma_{\mathrm{URA}}^{2}+\sigma_{\text {Iono }}^{2}+\sigma_{\text {Tropo }}^{2}+\sigma_{\mathrm{mp}}^{2},
$$

where $\sigma_{\mathrm{URA}}^{2}$ is the user range accuracy (URA) related to the satellite ephemeris and clock, broadcast in the GPS navigation message [2] (for GLONASS, this parameter is set in order to take into account the ephemeris inaccuracy with respect to GPS), $\sigma_{\text {Iono }}^{2}$ is the accuracy related to ionosphere delay after Klobuchar model application (whose expression is in [11]), $\sigma_{\text {Tropo }}^{2}$ is the accuracy related to troposphere error after correction model application [11], and $\sigma_{\mathrm{mp}}^{2}$ is the accuracy related to multipath error [11].

The PR rate measurement accuracy is assumed inversely proportional to $\sin (\mathrm{El})$, where $\mathrm{El}$ is the satellite elevation. $\mathrm{PR}$ and $\mathrm{PR}$ rate weights are the inverse of their accuracies.

The optimization criterion of the LS is to minimize the sum of the squared residuals defined as

$$
\underline{r}=\underline{z}-H \cdot \underline{\hat{x}}
$$

where $\underline{z}$ is the measurements vector and $\underline{\hat{x}}$ is the state vector, estimated with the considered technique $[12,13]$.

\section{Reliability Test}

Reliability refers to the consistency of the results provided by a system; internal and external reliability are, respectively, the ability to detect gross errors and the effect of an undetected blunder on the solution [14].

The integrity of a navigation system is defined as the ability to provide timely warnings to users when the system should not be used $[2,5]$. Reliability monitoring is based on statistical test of the observation residuals, with the aim of detecting and excluding measurement errors; in this work, three different RAIM FDE techniques have been developed and their performance is evaluated in terms of RMS and maximum error, for the horizontal and vertical components, and reliable availability.

Before RAIM application, a check is performed to screen out bad geometries, which could imply erroneous detections; in this work, the WARP (Weighted-ARP) parameter, a generalization of the classical ARP $[5,15]$, is used as integrity geometry parameter and its expression is

$$
\text { WARP }=\text { WSlope }_{\max } \cdot \sqrt{T_{g}} \text {, }
$$


where WSlope ${ }_{\max }$ is the maximum of the weighted slope [5, 16], which is an extension of the classical slope and takes into account the measurement different accuracies, and $T_{g}$ is the threshold of the global test and will be discussed shortly.

The FDE schemes analysed are obtained by combining different basic statistical tests. Specifically a so-called global test (GT) is adopted to verify the self-consistency of the measure set; if the measurement set is declared inconsistent, a local test (LT) is performed to identify and reject a blunder, after a separability check. Separability refers to the ability to separate any two measurements from one another [14]; this concept is primary to avoid the incorrect exclusion of blunder-free measurements.

In the first test the decision variable $D$ is defined as the sum of the squares of the residuals, weighted by the weighting matrix $W$ (consisting of the inverse of the squared measurement accuracies (9) on the diagonal and of zeros outside the diagonal):

$$
D=\underline{r}^{T} W \underline{r},
$$

where $D$ is assumed to follow a $\chi^{2}$ distribution with $(m-n)$ degrees of freedom or redundancy, defined as the difference between the number of measurements $m$ and state dimension $n$; an inconsistency in the observations is assumed if $D$ exceeds the threshold $T_{g}$ :

$$
T_{g}=\chi_{1-P_{\mathrm{FA}},(m-n)}^{2},
$$

where the notation $\chi_{1-P_{\mathrm{FA}},(m-n)}^{2}$ indicates the abscissa corresponding to a probability value $\left(1-P_{\mathrm{FA}}\right)$ of a $\chi^{2}$ distribution of $(m-n)$ order. $P_{\mathrm{FA}}$ is the probability of false alarm and is fixed in accordance with application requirements; $P_{\mathrm{FA}}$ typical value for urban navigation is $0.1 \%$ [17] while $T_{g}$ varies with redundancy.

If the GT does not pass, a LT is performed analyzing standardized residual $w_{i}[18]$ :

$$
w_{i}=\left|\frac{r_{i}}{\sqrt{\left(C_{r}\right)_{i i}}}\right|, \quad i=1: m,
$$

where $C_{r}$ is the residuals variance covariance matrix.

$w_{i}$ are assumed to be normally distributed; each element $w_{i}$ that exceeds the local threshold $T_{l}$, corresponding to the probability value $\left(1-P_{\mathrm{FA}} / 2\right)$ of a normal distribution, is flagged as blunder. The measurement corresponding to the largest standardized residual exceeding $T_{l}$ is excluded or deweighted (depending on the method) after a separability check; a large blunder could cause multiple local test failures and therefore an erroneous measurement rejection. In RAIM techniques, a parameter properly representing the separability is the correlation coefficient of $w_{i}$, shown in the following:

$$
\gamma_{i j}=\frac{\left(C_{r}\right)_{i j}}{\sqrt{\left(C_{r}\right)_{i i} \cdot\left(C_{r}\right)_{j j}}}, \quad i, j=1: m .
$$

In the separability check, if $\gamma_{i j}$ exceeds a threshold (in this case 0.9), the measurement suspected to be a blunder is not rejected or deweighted, because it is strictly correlated to another measurement.

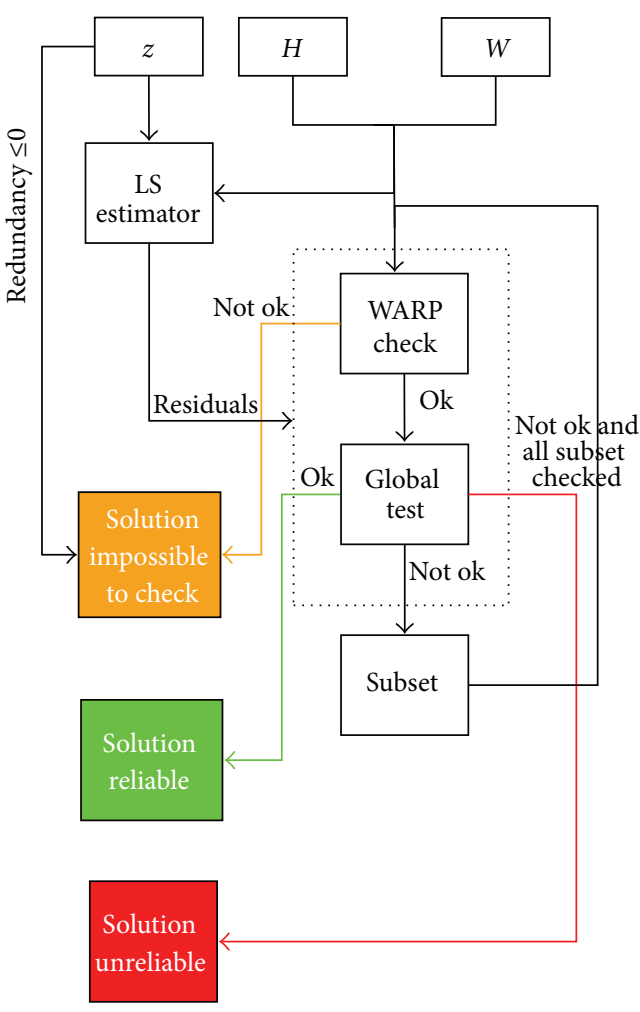

FIgURE 1: Subset algorithm.

3.1. Subset. Subset testing is an FDE technique that uses only GT $[5,19,20]$. If a measurements set is declared inconsistent, all the possible combinations of measurements are checked, to find a subset from which the supposed blunders are excluded. Only the subset that passes the GT and is declared consistent is used to compute the navigation solution; if more subsets pass the GT, the set with the minimum statistic variable and the largest number of measurements is chosen. In this technique the separability check is not performed, because standardized residuals are not analysed. A complete scheme of the algorithm implemented is shown in Figure 1.

The Subset test is applied separately to pseudorange and Doppler measurements and is computationally heavy because several measurement combinations have to be checked [20].

3.2. Forward-Backward. Forward-Backward is an FDE technique that involves the use of both global and local tests; it consists of two different steps [20-22]. The first algorithm section, called Forward, is carried out to identify and exclude erroneous measurements. A measurement set is preliminary tested for the integrity geometry to screen out bad geometries which could imply erroneous detections. After the preliminary check, the GT is carried out in order to verify measurements consistency. If global test declares the set inconsistent, the LT is performed to identify and exclude the erroneous measurement. An erroneous rejection of a good observation is possible, due to the mutual influence of the 


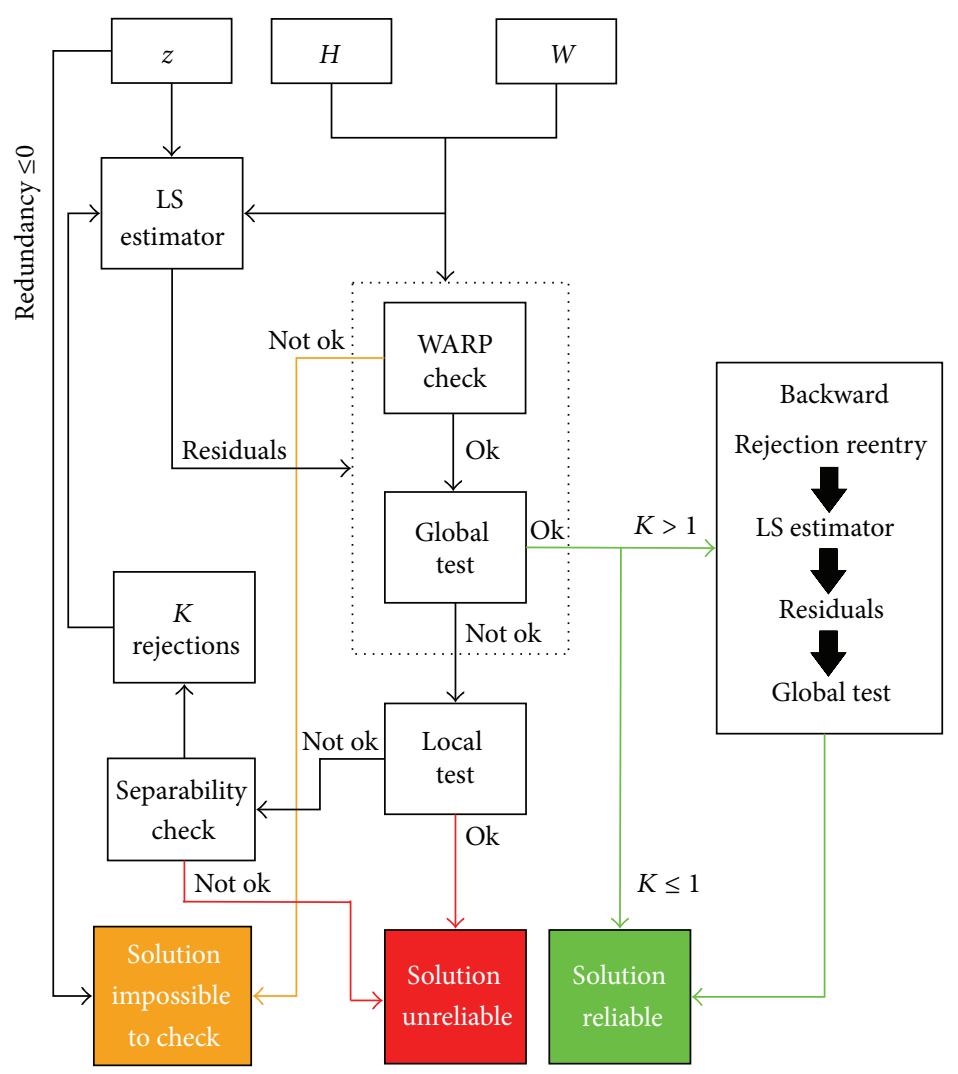

FIGURE 2: Forward-Backward algorithm.

observations; to avoid erroneous rejections, the separability check is carried out: the measurement flagged as possible blunder is excluded only if it is not correlated with other measures. Forward process is performed recursively until no more erroneous measurements are found and the solution is declared reliable or unreliable.

If the solution is declared reliable and $k$ measurements are excluded (with $k>1$ ), the Backward scheme is applied to reintroduce observations wrongly excluded. Rejected measurements are iteratively implemented backward and the global test is performed; the observation set which passes the GT is used to compute navigation solution.

A complete scheme of the Forward-Backward technique is shown in Figure 2.

3.3. Danish. Least squares estimation is very susceptible to outliers; a possible way to solve this problem is an iterative deweighing of erroneous measurements [20,23]. The Danish method is an iteratively reweighted least squares algorithm, used in geodetic applications for a long time; this method is used to achieve consistency between the measurements by modifying the a priori weights. In this paper, the Danish method is used for signal degraded environments in order to minimize the effect of blunders on the least square adjustment. This technique involves the use of the GT, to verify the consistency of the measurements, and the LT, to identify and deweight the outliers.
A measurement set is checked for the geometry, as in the previous FDE techniques and then the GT is performed. If observations are declared not consistent by GT, the LT is carried out to identify the blunder, and its related weight is reduced only if allowed by the separability check (i.e., if the measurement is not strictly correlated to the others). The variance of the suspected measurement is exponentially increased (and consequently the weight is decreased) as follows:

$$
\sigma_{i, j+1}^{2}=\sigma_{i, 0}^{2} * \begin{cases}e^{w_{i, j} / T_{l}} & \text { if } w_{i, j}>T_{l} \\ 1 & \text { if } w_{i, j} \leq T_{l}\end{cases}
$$

where $\sigma_{i, j+1}^{2}$ is the variance of the $i$ th observation after $j+1$ iterations, $\sigma_{i, 0}^{2}$ is the a priori variance of the observation, and $w_{i, j}$ is the standardized residual of the $i$ th observation after $j$ iterations.

If the normalized residual of the $i$ th observation does not exceed $T_{l}$, its variance is maintained (the measurement is not deweighted).

The scheme of the Danish procedure is shown in Figure 3.

\section{Test and Results}

4.1. Test. A static test of about 6 hours was carried out on February 24, 2012. The antenna was placed on the roof of the 


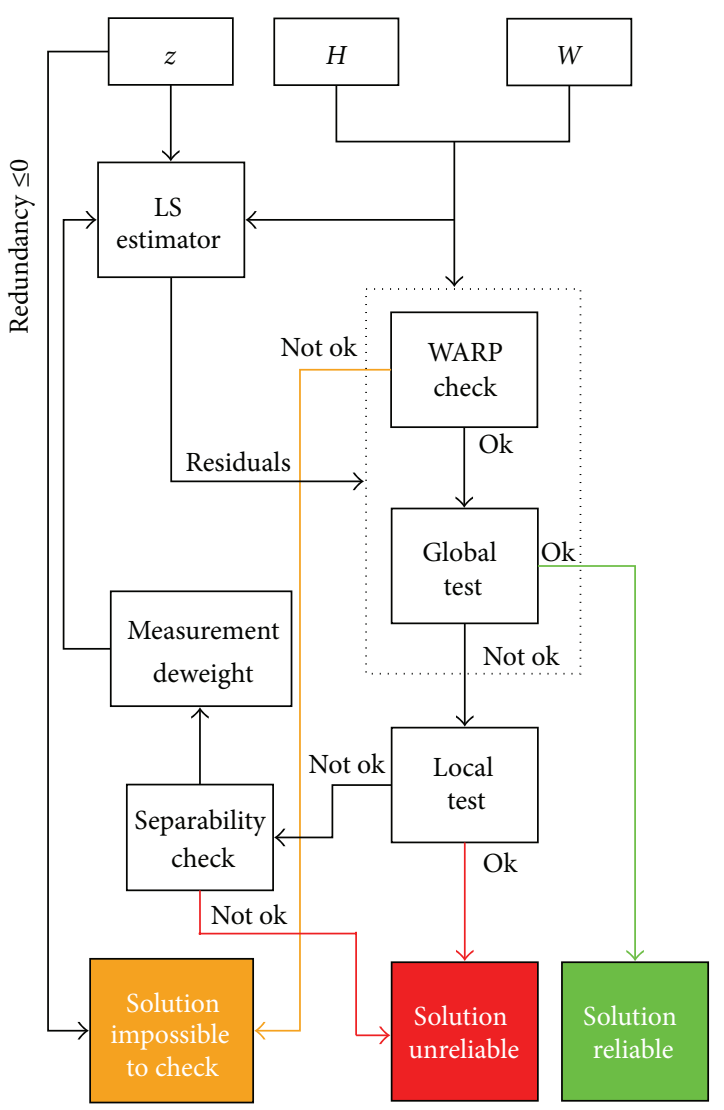

FIGURE 3: Danish algorithm.

PANG (PArthenope Navigation Group) laboratory building, Centro Direzionale of Naples (Italy), a typical example of urban canyon (as shown in Figure 4); in this environment many GNSS signals are blocked by skyscrapers or are strongly degraded for the multipath phenomenon. The only test performed for this research was static to simplify the error analysis for the position (the antenna is placed in a wellknown location) and for the velocity (the antenna is fixed, so its velocity is zero); a kinematic test needs a reference for error analysis more complicated to obtain and will be performed in the future develop of this research. However, the static test choice does not limit the research validity because the operational environment is a typical signaldegraded scenario, that is, an urban canyon.

The used receiver is a NovAtel FlexPak-G2, able to provide single frequency (L1) GPS/GLONASS measurements, connected to a NovAtel antenna 702-GG.

The reference solution is computed by a postprocessing geodetic method, guaranteeing a position accuracy of $\mathrm{mm}$ order; the coordinates of the antenna and the relative accuracies are shown in Table 2.

4.2. Results. Herein eight different GNSS configurations are compared, combining the two systems considered and the different RAIM scheme developed:

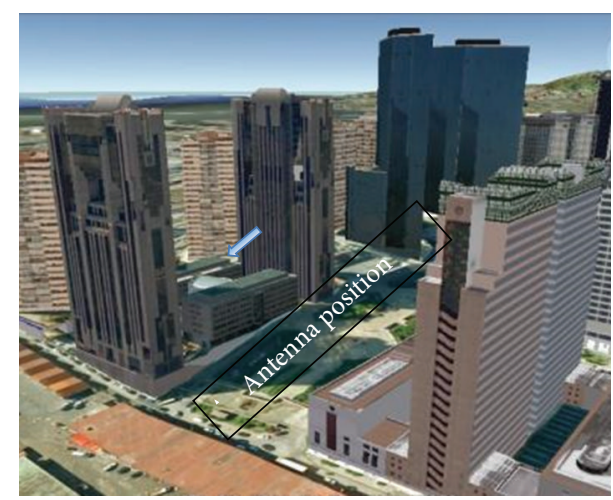

Figure 4: Antenna position.

(i) GPS only without RAIM application (briefly indicated as GPS no RAIM);

(ii) GPS/GLONASS without RAIM application (GG no RAIM);

(iii) GPS only with Subset RAIM application (GPS Sub);

(iv) GPS/GLONASS with Subset RAIM application (GG Sub);

(v) GPS only with Forward-Backward RAIM application (GPS FB);

(vi) GPS/GLONASS with Forward-Backward RAIM application (GG FB);

(vii) GPS only with Danish method applied (GPS Dan);

(viii) GPS/GLONASS with Danish method applied (GG Dan).

Results are analyzed in terms of RMS and maximum errors for horizontal and vertical components in the position and velocity domains. The percentage of time mission when solution is available is referred to as solution availability; in case of RAIM application, the concept of reliable availability, defined as the time percentage when solution is reliable, is introduced.

The session is characterized by high solution availability (about 98\% for GPS and 100\% GPS/GLONASS configuration) and by very large errors (more than $1 \mathrm{~km}$ without RAIM application); the application of the developed RAIM schemes reduces the availability of the position solution. In GPS stand-alone configuration, the Subset test guarantees the highest reliable availability (76.2\%), while $20 \%$ of solutions are flagged as unreliable; for Danish and Forward-Backward schemes, the reliable availability is halved with respect to the solution availability. The inclusion of GLONASS measurements, without RAIM application, improves the solution availability of $2 \%$ with respect to the GPS stand-alone configuration. For the GPS/GLONASS multiconstellation, the Subset testing guarantees an high reliable availability (only $3.5 \%$ of solutions are rejected by the quality control) which is increased to $20 \%$ with respect to the GPS only configuration. The effect of the GLONASS inclusion is more evident in the 


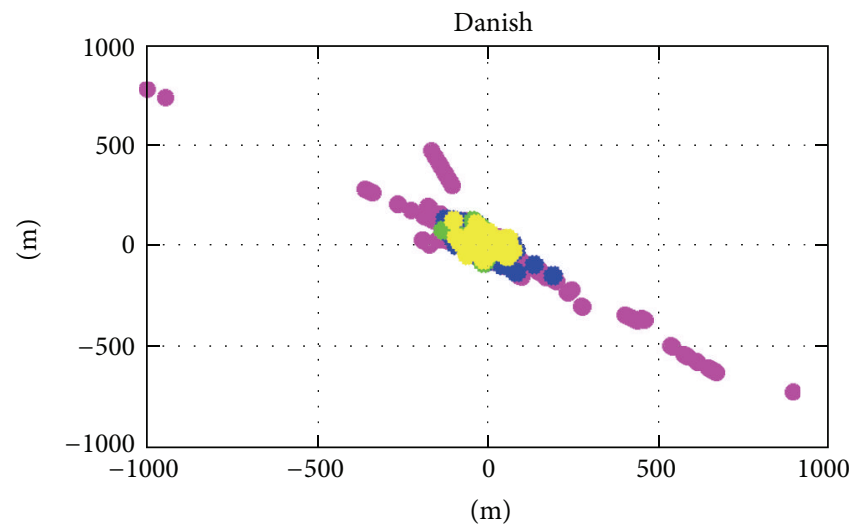

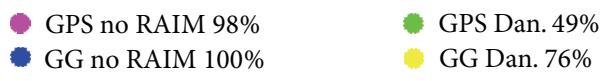

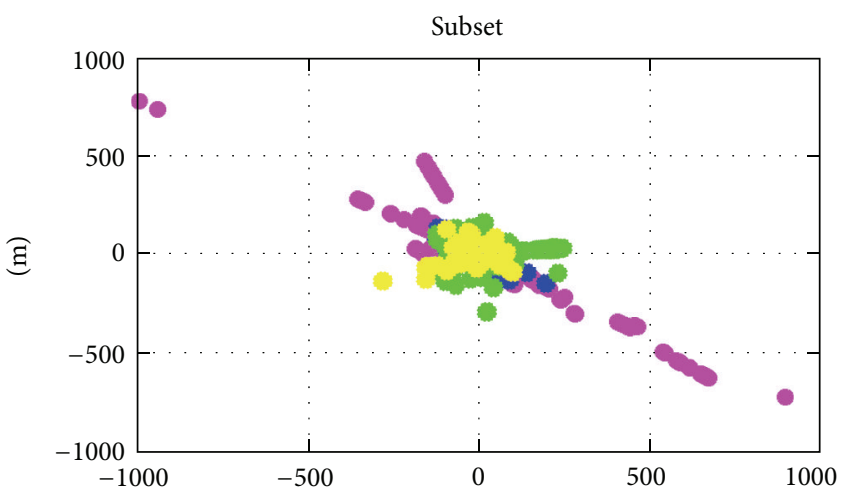

$(\mathrm{m})$
GPS no RAIM $98 \%$ GPS Sub. $76 \%$
GG Sub. $97 \%$

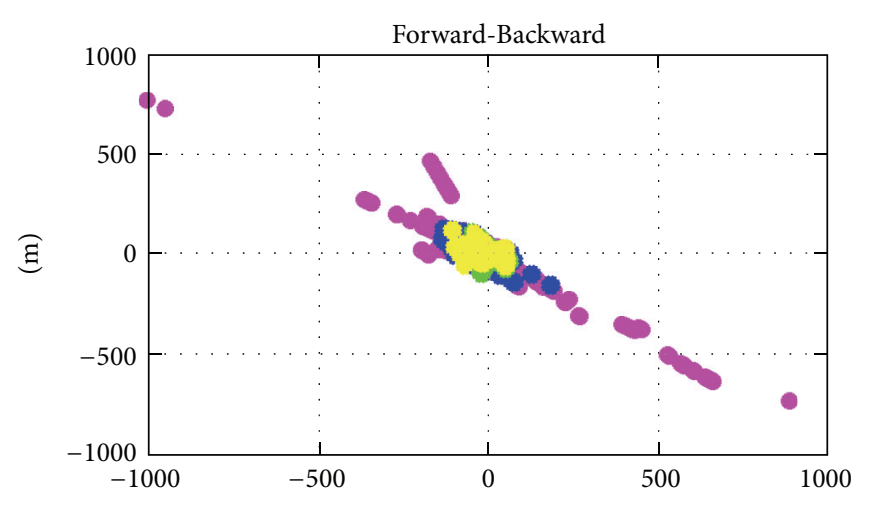

(m)

- GPS no RAIM 98\% GPS FB $44 \%$

- GG no RAIM 100\% GG FB 74\%

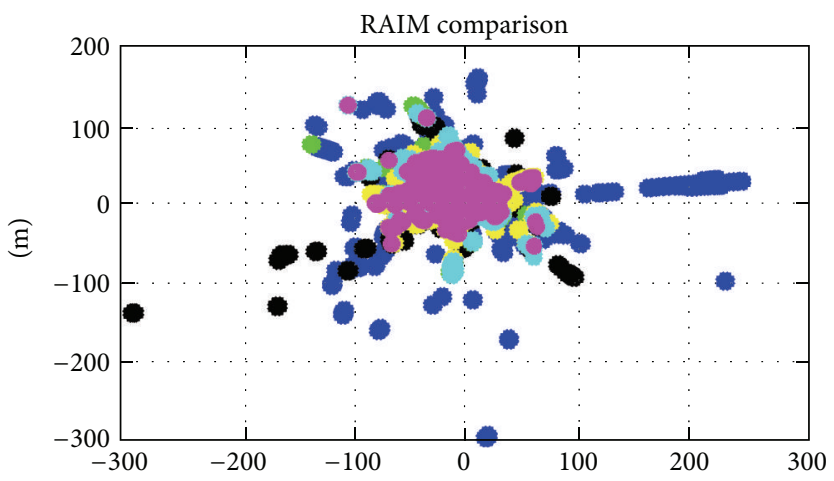

(m)

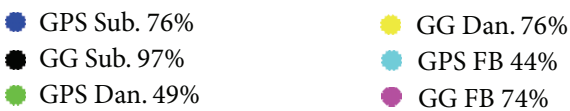

Figure 5: Horizontal position.

TABLE 2: PANG station coordinates and accuracy.

\begin{tabular}{lccccc}
\hline $\begin{array}{l}\text { Latitude } \\
(\mathrm{deg}, \mathrm{min}, \mathrm{sec})\end{array}$ & $\begin{array}{c}\text { Longitude } \\
(\mathrm{deg}, \mathrm{min}, \mathrm{sec})\end{array}$ & $\begin{array}{c}\text { Height } \\
(\mathrm{m})\end{array}$ & $\begin{array}{c}\text { Standard deviation north } \\
(\mathrm{m})\end{array}$ & $\begin{array}{c}\text { Standard deviation east } \\
(\mathrm{m})\end{array}$ & $\begin{array}{c}\text { Standard deviation up } \\
(\mathrm{m})\end{array}$ \\
\hline $40^{\circ} 51^{\prime} 23.5163^{\prime \prime}$ & $14^{\circ} 17^{\prime} 03.8997^{\prime \prime}$ & 90.6257 & 0.0006 & 0.0008 & 0.0011 \\
\hline
\end{tabular}

Danish and Forward-Backward schemes; in these cases the reliable availability reaches about $75 \%$ with an improving of $25-30 \%$ with respect to the GPS only configurations. The solution availability and the reliable availability of the position are summarized in Table 3.

Similar results are obtained in velocity domain; the GLONASS measurements increase the reliable availability for the three RAIM schemes relative to GPS only configurations; as in the position domain, the Subset testing guarantees higher reliable availability with respect to the other developed schemes. The solution availability and the reliable availability of the velocity are summarized in Table 4 .

The configurations without RAIM are characterized by large errors: in case of GPS only, the maximum horizontal error exceeds $1 \mathrm{~km}$ and the inclusion of GLONASS measurements improves the performance reducing the maximum error to 245 meter. RAIM application improves all considered configurations, reducing both maximum and RMS errors, as shown in Figure 5 for the horizontal component; in the upper figures and in the bottom left one, the performance of RAIM schemes is compared with the basic configurations (no RAIM), and in all cases the clouds relative to the RAIM solutions are significantly reduced with respect to the baseline configurations. In the bottom right of Figure 5, the performances of the three developed RAIM schemes are compared; from a qualitative analysis the cloud relative to the GPS/GLONASS with forward-backward scheme is more concentrated with respect to the others. 

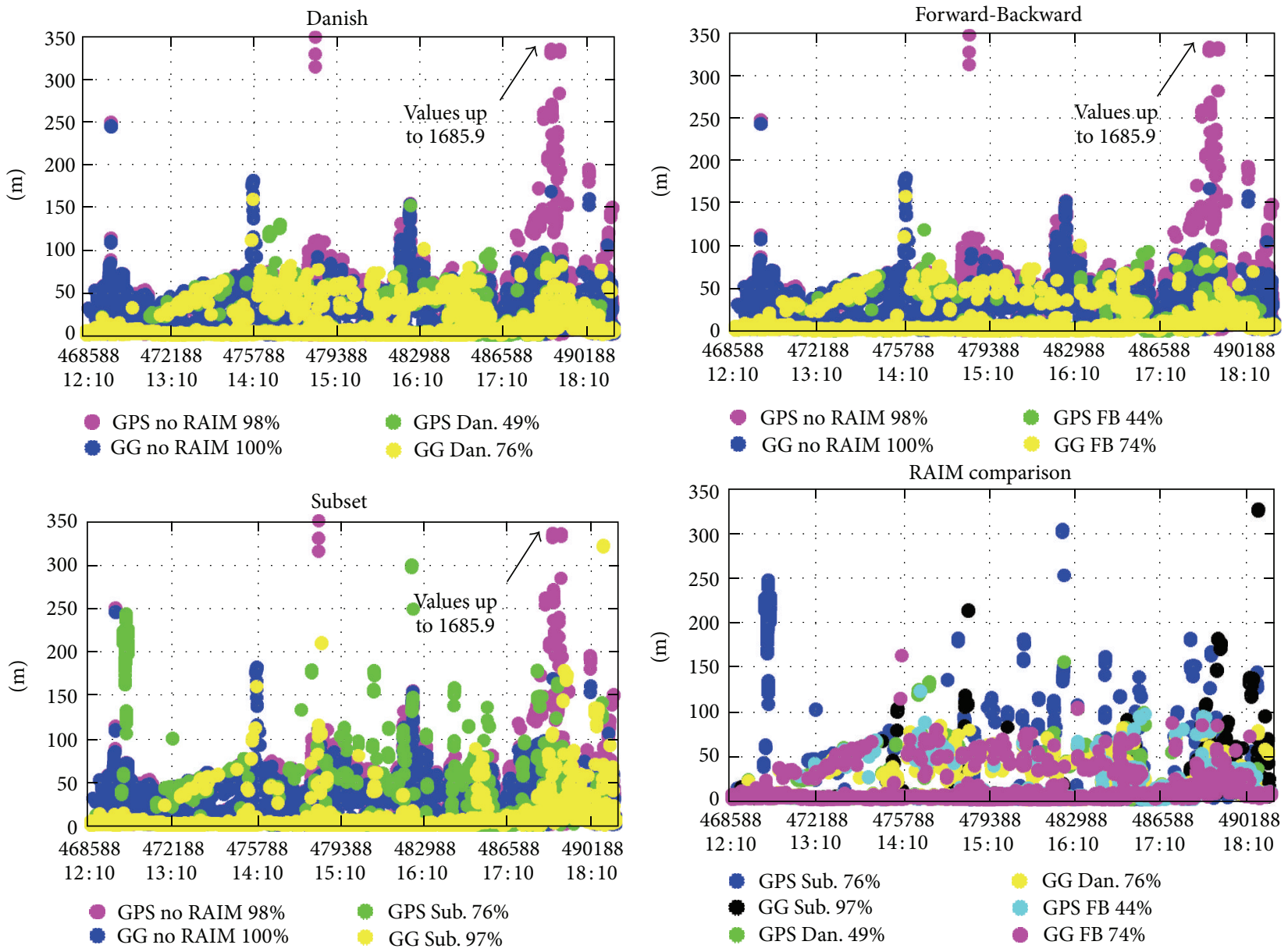

FIgURE 6: Horizontal position error.

TABLE 3: Position availability/reliable availability.

\begin{tabular}{lcccc}
\hline & $\begin{array}{c}\text { No RAIM } \\
\text { availability \% }\end{array}$ & $\begin{array}{c}\text { Subset } \\
\text { reliable availability \% }\end{array}$ & $\begin{array}{c}\text { Danish } \\
\text { reliable availability \% }\end{array}$ & $\begin{array}{c}\text { Forward-Backward } \\
\text { reliable availability \% }\end{array}$ \\
\hline GPS & 98.1 & 76.2 & 49.0 & 43.6 \\
GG & 100 & 96.5 & 75.8 & 74.0 \\
\hline
\end{tabular}

In Figure 6 the horizontal position errors are plotted. In the upper figures and in the bottom left one, the errors of the three RAIM schemes are compared with the "no RAIM" configurations; the errors of the RAIM configurations, represented by the green and yellow dots, are lower than the errors of the no RAIM configurations confirming the aforesaid qualitative analysis. In the bottom right of Figure 6, the RAIM errors are compared; the Subset testing is characterized by the highest reliable availability but also by the highest errors, while the other schemes provide similar performance. Also in these cases the benefits of the inclusion of GLONASS measurements are clear; all the GPS/GLONASS configurations, represented by the black, yellow, and magenta dots, are lower with respect to the corresponding GPS only configurations. The GLONASS measurements improve the redundancy increasing the RAIM efficiency.

In Figure 7 the horizontal velocity errors are plotted; "no RAIM" configurations are characterized by high error; for GPS only case higher than $50 \mathrm{~m} / \mathrm{s}$, the GLONASS inclusion reduces the errors up to $0.44 \mathrm{~m} / \mathrm{s}$. As done for the position errors, the RAIM schemes are first compared with the "no RAIM" configurations; in the velocity domain the benefits of the RAIM application are less evident with respect to the position domain due to the robustness of the Doppler observable. In the bottom right part of Figure 7, the horizontal velocity errors obtained with the FDE techniques are compared; as in 

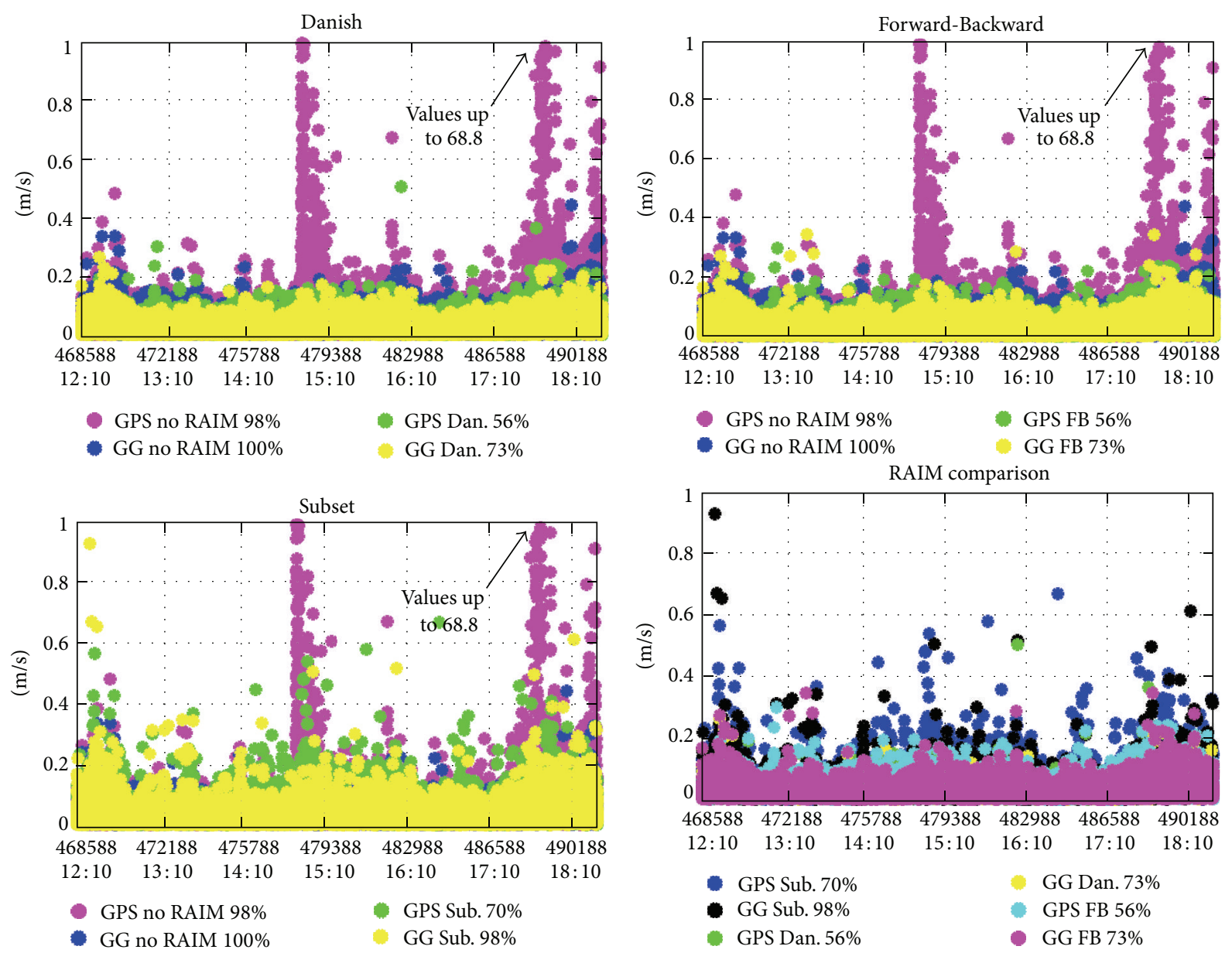

FIGURE 7: Horizontal velocity error.

the position domain, the Subset testing is characterized by the highest reliable availability but also by the highest errors, the other schemes provide similar performance.

In Figures 8 and 9, the plots relative to the vertical components of the position and velocity show the benefits of RAIM use.

The qualitative analysis, provided by the previous plots, is confirmed by the results summarized in Tables 5 and 6; in detail, the RAIM use improves significantly the performance in terms of RMS and maximum errors for the vertical and horizontal components, maintaining a high reliable availability in case of GG configurations. The RMS horizontal errors are halved and even better results are evident on the vertical component. The maximum horizontal and vertical errors for GG Subset configuration are degraded due to an erroneous measurements rejection in the presence of multiple blunders; better results are obtained with the Forward-Backward and Danish methods.

\section{Conclusions}

In signal-degraded environments such as urban canyons, GNSS navigation suffers the presence of gross errors which strongly worsen the solution; therefore, in these scenarios the use of RAIM algorithms is necessary. In this work three RAIM FDE schemes, well known in literature [17, 18, 20-23], are adopted: Subset testing, Forward-Backward, and Danish. The first method is based uniquely on the GT, while the others include the use of the LT too. These methods have been enriched adopting a preliminary check on the satellite geometry, to screen out configurations too poor to be tested successfully, and a separability test, to avoid the exclusion of blunder-free measurements in case of observations strongly correlated. The main scope of this work is to verify the effectiveness of the RAIM schemes with the proposed modifications in urban scenario. Moreover, the benefit assessment of GLONASS inclusion is of interest too.

The obtained results show the effectiveness of the adopted algorithms in terms of reliable availability and of RMS and maximum errors. The reliable availability is the percentage of time mission when the solution is declared reliable by the adopted RAIM; the highest value of this parameter is obtained with the Subset method, which provides the largest errors too. The Forward-Backward and the Danish methods are instead characterized by similar performances and by the smallest errors, demonstrating the validity of the separability 

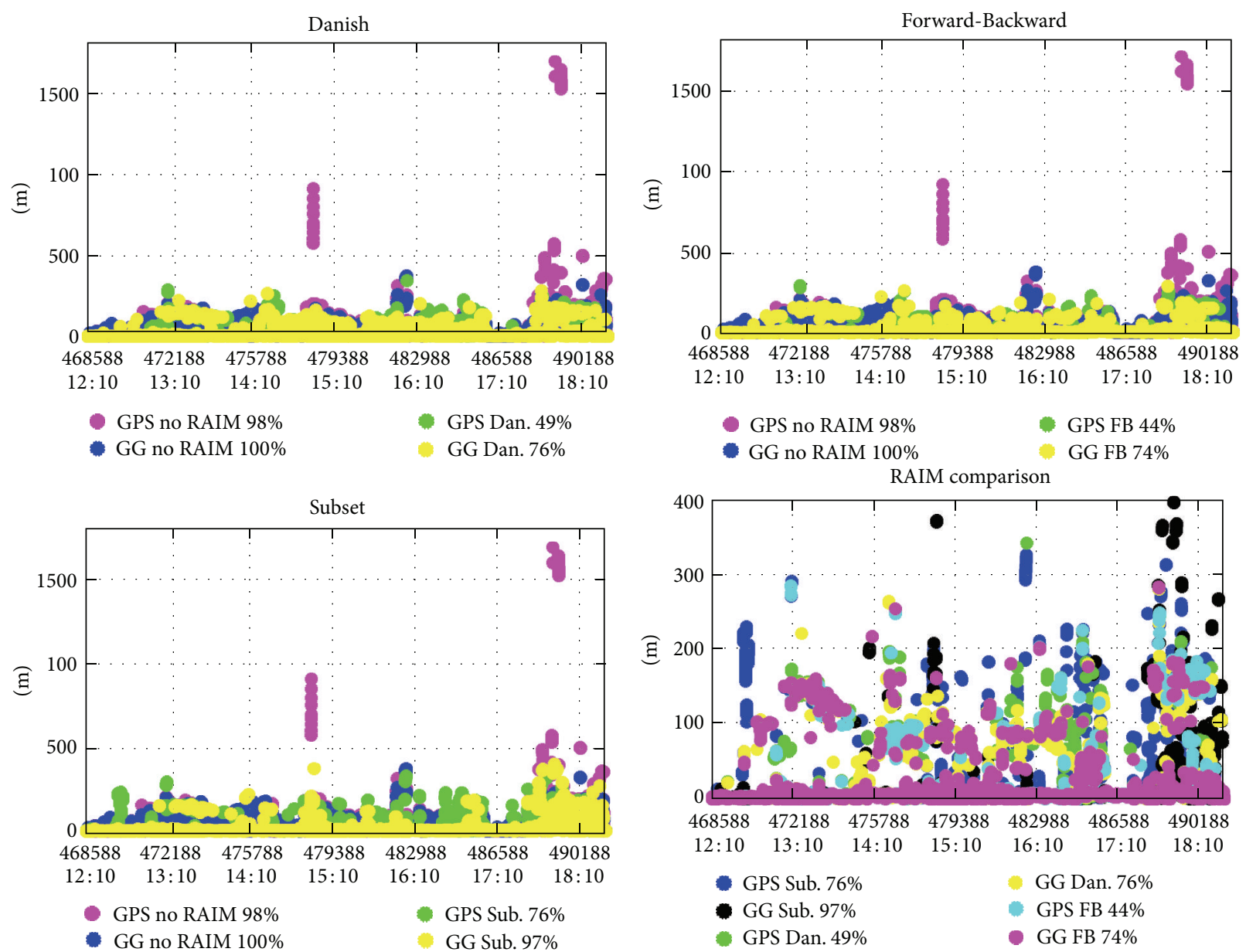

Figure 8: Altitude.

TABLE 4: Velocity availability/reliable availability.

\begin{tabular}{lcccc}
\hline & $\begin{array}{c}\text { No RAIM } \\
\text { availability } \%\end{array}$ & $\begin{array}{c}\text { Subset } \\
\text { reliable availability } \%\end{array}$ & $\begin{array}{c}\text { Danish } \\
\text { reliable availability \% }\end{array}$ & $\begin{array}{c}\text { Forward-backward } \\
\text { reliable availability \% }\end{array}$ \\
\hline GPS & 98.1 & 70.5 & 56.3 & 56.3 \\
GG & 100 & 97.8 & 73.4 & 72.9 \\
\hline
\end{tabular}

TABLE 5: Position results.

\begin{tabular}{lccccc}
\hline & & \multicolumn{2}{c}{ RMS (m) } & \multicolumn{2}{c}{ MAX (m) } \\
& & $H$ & $V$ & $H$ & $V$ \\
\hline \multirow{2}{*}{ No RAIM } & GPS & 54.9 & 85.6 & 1264.5 & 1685.9 \\
Subset & GG & 34.8 & 65.4 & 245.6 & 372.2 \\
\multirow{3}{*}{ Forward-backward } & GPS & 27.5 & 56.4 & 299.0 & 327.0 \\
\multirow{4}{*}{ Danish } & GG & 15.1 & 36.1 & 321.6 & 398.5 \\
& GG & 17.9 & 44.5 & 159.7 & 285.8 \\
& GPS & 13.4 & 31.3 & 159.7 & 284.3 \\
& GG & 16.0 & 56.1 & 159.7 & 343.1 \\
& & & & & \\
\hline
\end{tabular}

TABLE 6: Velocity results.

\begin{tabular}{lccccc}
\hline & & \multicolumn{2}{c}{ RMS (m/s) } & \multicolumn{2}{c}{ MAX (m/s) } \\
& & $H$ & $V$ & $H$ & $V$ \\
\hline \multirow{2}{*}{ No RAIM } & GPS & 0.968 & 1.573 & 68.750 & 108.240 \\
Subset & GG & 0.042 & 0.060 & 0.442 & 0.822 \\
Forward-Backward & GPS & 0.053 & 0.084 & 0.669 & 1.251 \\
& GG & 0.042 & 0.067 & 0.928 & 1.337 \\
Danish & GG & 0.047 & 0.074 & 0.649 & 1.251 \\
& GPS & 0.036 & 0.055 & 0.367 & 0.653 \\
& GG & 0.035 & 0.073 & 0.649 & 1.251 \\
& & & & & \\
\hline
\end{tabular}



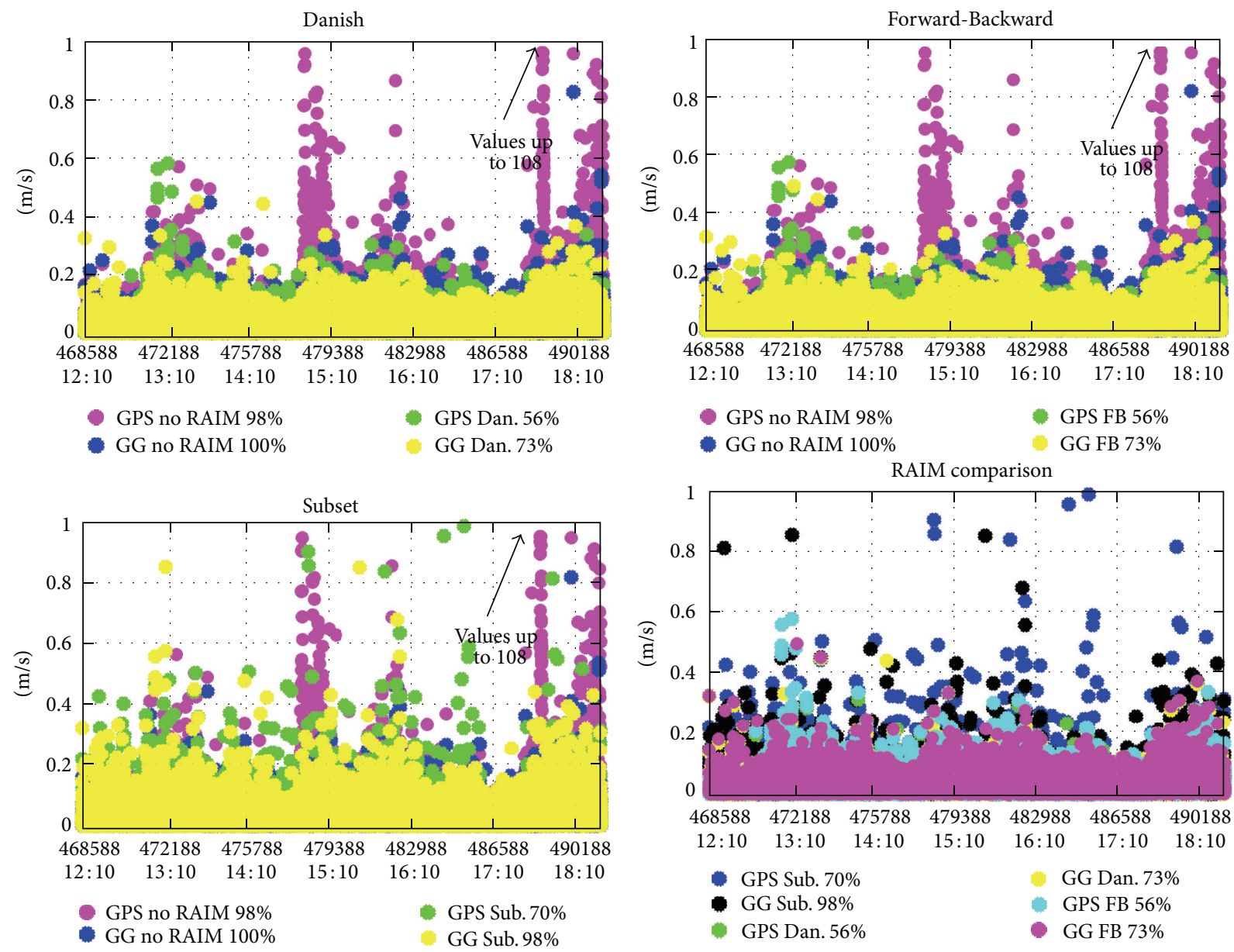

FIgURE 9: Vertical velocity error.

check module (which cannot be applied to Subset method). The proposed algorithms have been tested on both position and velocity domains, showing comparable robustness.

GPS/GLONASS combination shows evident performance improvements, for all the considered parameters, relative to GPS only configurations.

\section{References}

[1] B. Hoffmann-Wellenhof, H. Lichtenegger, and J. Collins, Global Positioning System: Theory and Practice, Springer, New York, NY, USA, 1992.

[2] E.D. Kaplan and J. Hegarty, "Fundamentals of satellite navigation," in Understanding GPS: Principles and Applications, E. D. Kaplan, Ed., Artech House Mobile Communications Series, 2nd edition, 2006.

[3] A. Angrisano, M. Petovello, and G. Pugliano, "GNSS/INS integration in vehicular urban navigation," in Proceedings of the 23rd International Technical Meeting of the Satellite Division of the Institute of Navigation (GNSS '10), pp. 1505-1512, The Institute of Navigation, Portland, Ore, USA, September 2010.

[4] A. Angrisano, M. Petovello, and G. Pugliano, "Benefits of combined GPS/GLONASS with low-cost MEMS IMUs for vehicular urban navigation," Sensors, vol. 12, no. 4, pp. 51345158, 2012.
[5] B. Parkinson and J. J. Spilker, Global Positioning System: Theory And Applications, vol. 1-2, American Institute of Aeronautics and Astronautics, Washington, DC, USA, 1996.

[6] C. Cai, Precise point positioning using dual-frequency GPS and GLONASS measurements [M.S. thesis], UCGE Report no. 20291, Department of Geomatics Engineering, University of Calgary, Calgary, Canada, 2009.

[7] A. Angrisano, GNSS/INS integration methods [Ph.D. thesis], "Parthenope" University of Naples, 2010.

[8] C. Cai and Y. Gao, "A combined GPS/GLONASS navigation algorithm for use with limited satellite visibility," Journal of Navigation, vol. 62, no. 4, pp. 671-685, 2009.

[9] A. Angrisano, S. Gaglione, G. Pugliano, R. Robustelli, R. Santamaria, and M. Vultaggio, "A stochastic sigma model for GLONASS satellite pseudorange," Applied Geomatics, vol. 3, no. 1, pp. 49-57, 2011.

[10] ICD-GLONASS, Global Navigation Satellite System GLONASS Interface Control Document, version 5.1, Moscow, Russia, 2008.

[11] SC-159 of the RTCA, Minimum Operational Performance Standards for Global Positioning System/Wide Area Augmentation System Airborne Equipment, Document DO-229D, RTCA, Washington, DC, USA, 2006.

[12] E. M. Mikhail, Observations and Least Squares, Harper \& Row, 1976. 
[13] D. E. Wells and E. J. Krakiwsky, The Methods of Least Squares, Lecture Notes no 18, Department of Surveying Engineering, University of Brunswick, 1971.

[14] S. Hewitson and J. Wang, "GNSS receiver autonomous integrity monitoring (RAIM) performance analysis," GPS Solutions, vol. 10, no. 3, pp. 155-170, 2006.

[15] G. Y. Chin, J. H. Kraemer, and R. G. Brown, "GPS RAIM: screening out bad geometries under worst-case bias conditions," Navigation, Journal of the Institute of Navigation, vol. 39, no. 4, pp. 407-427, 1992.

[16] R. G. Brown and G. Y. Chin, "GPS RAIM: calculation of threshold and protection radius using chi-square methodsa geometric approach," Global Positioning System: Institute of Navigation, vol. 5, pp. 155-179, 1997.

[17] M. Petovello, Real-time integration of a tactical-grade IMU and GPS for high-accuracy positioning and navigation [Ph.D. thesis], UCGE Report no. 20173, Department of Geomatics Engineering, University of Calgary, Calgary, Canada, 2003.

[18] W. Baarda, A Testing Procedure for Use in Geodetic Networks, Netherlands Geodetic Commission, Publication on Geodesy, New Series 2, 5, Delft, The Netherlands, 1968.

[19] A. K. Brown, "Receiver autonomous integrity monitoring using a 24-satellite GPS constellation," Navigation, Journal of The Institute of Navigation, vol. 5, pp. 21-33, 1998, Red Book of RAIM.

[20] H. Kuusniemi, User-level reliability and quality monitoring in satellite-based personal navigation [Ph.D. thesis], Tampere University of Technology, Tampere, Finland, 2005.

[21] H. Kuusniemi, A. Wieser, G. Lachapelle, and J. Takala, "User-level reliability monitoring in urban personal satellitenavigation," IEEE Transactions on Aerospace and Electronic Systems, vol. 43, no. 4, pp. 1305-1318, 2007.

[22] A. Angrisano, S. Gaglione, and C. Gioia, "RAIM algorithms for aided GNSS in urban scenario," in Proceedings of the Ubiquitous Positioning Indoor Navigation and Location Based Service, Helsinki, Finland, October 2012.

[23] A. Leick, GPS Satellite Surveying, John Wiley \& Sons, Hoboken, NJ, USA, 3rd edition, 2004. 

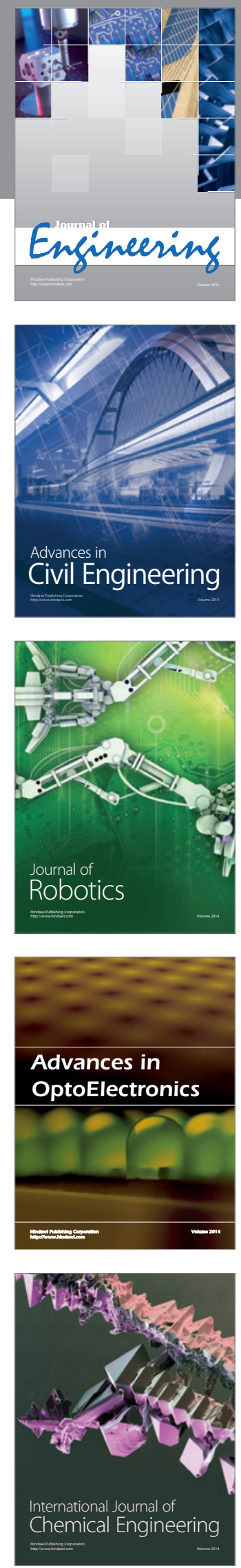

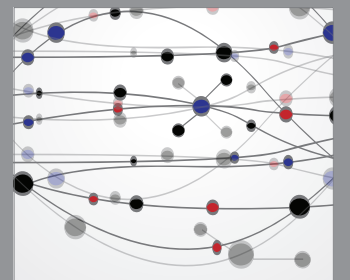

The Scientific World Journal
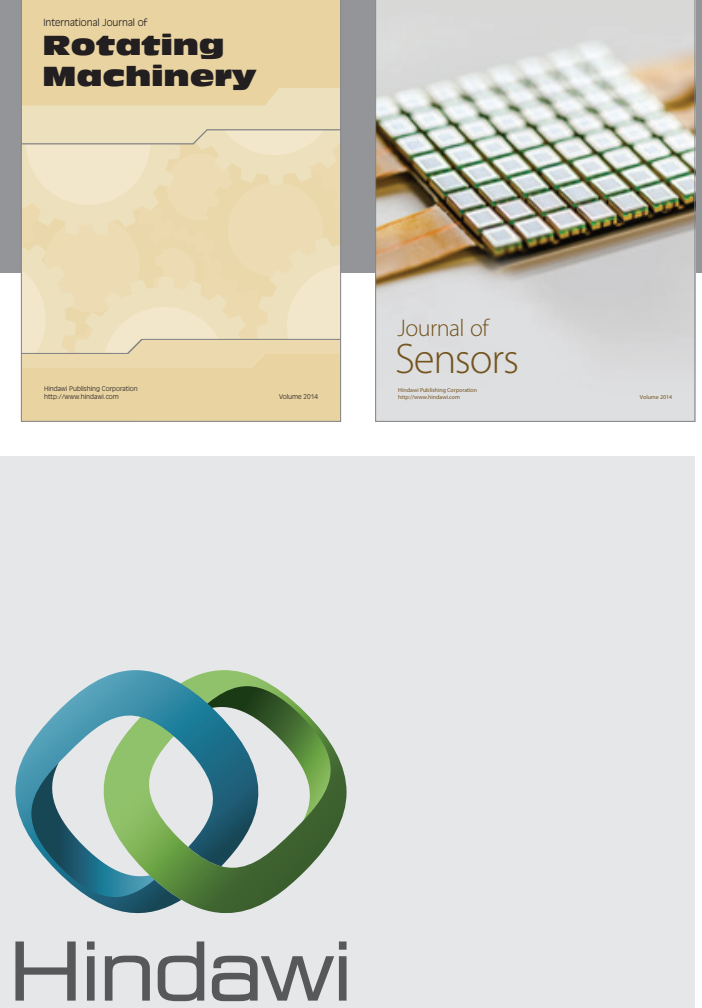

Submit your manuscripts at http://www.hindawi.com
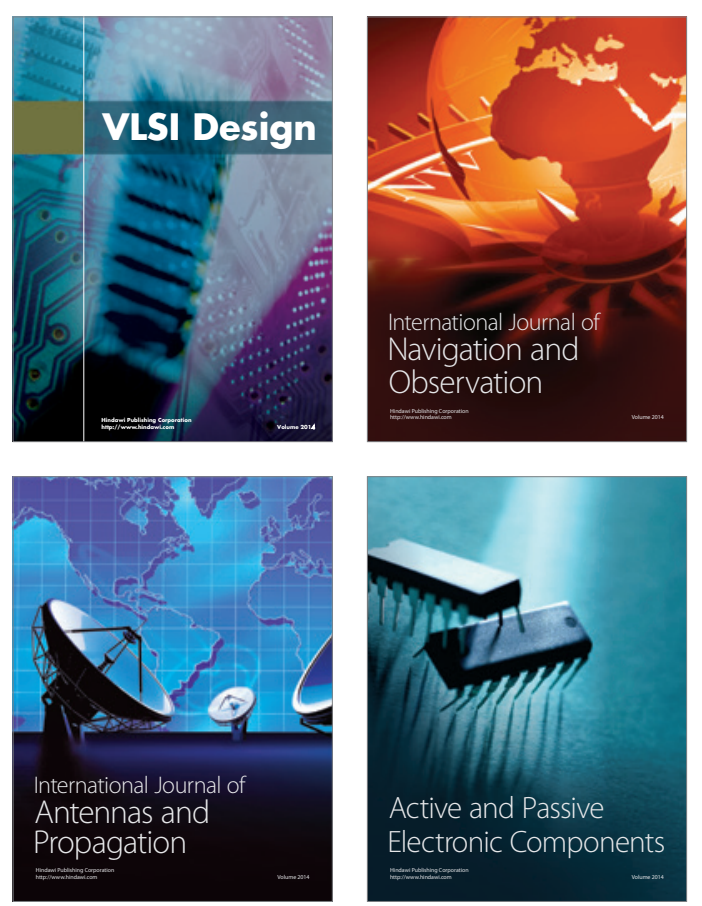
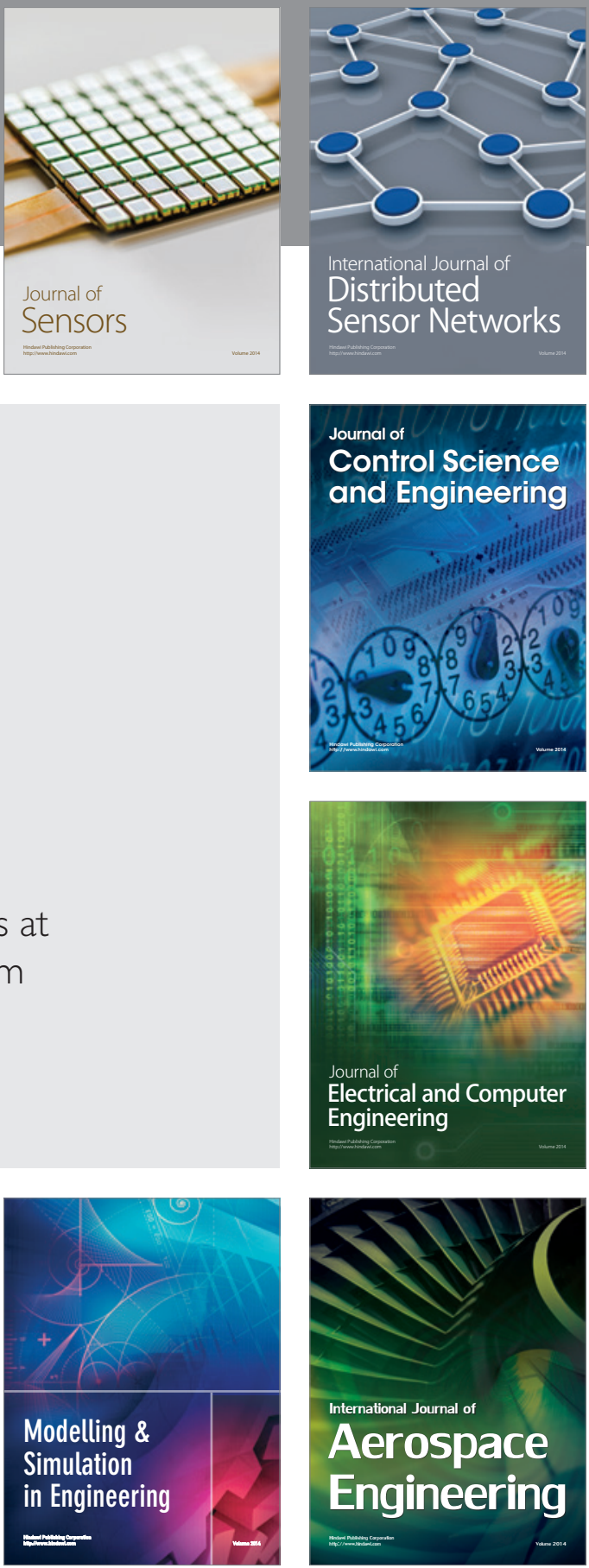

Journal of

Control Science

and Engineering
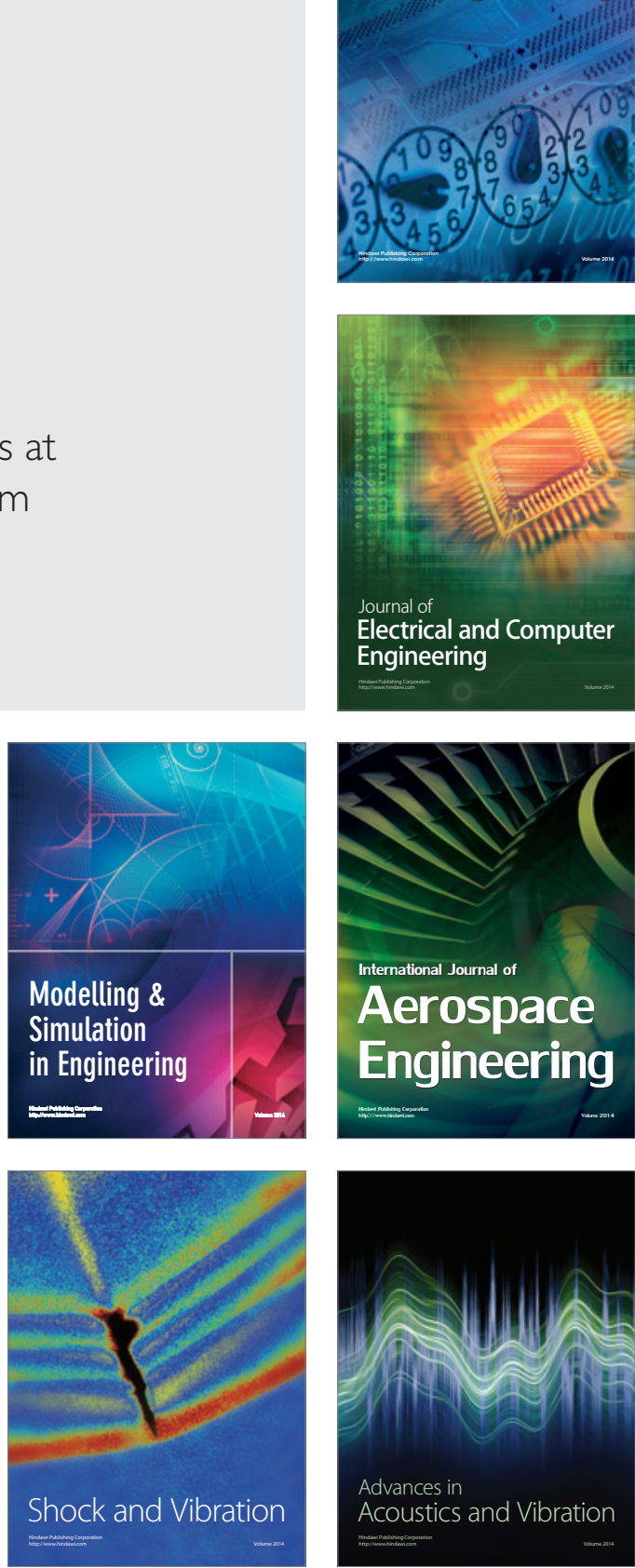